\title{
Evolución del tamaño de la vivienda de promoción pública y su comparación con el resto del parque residencial construido en Madrid entre 1940-2010
}

\author{
Evolution of the size of public housing and its comparison with the rest \\ of residential in Madrid between 1940-2010 \\ L. Moya González ${ }^{(*)}, \underline{\text { C. Fernández Salgado }}{ }^{(*)}$, F. Escamilla Valencia ${ }^{(*)}$
}

\section{RESUMEN}

Los valores cualitativos positivos de gran parte de la promoción pública residencial (VP) en Madrid han sido puestos de manifiesto en diferentes estudios, habida cuenta de la limitada economía de medios en su construcción. Sin embargo, una primera idea sobre la carestía de tamaño ha acompañado a la VP, identificándola como una vivienda pequeña. El artículo analiza si es así, si el parque que se conserva de VP en Madrid promovido desde 1940 y hasta 2010 resulta ser especialmente pequeño. Con ello se pretende conocer cuál ha sido la evolución de su tamaño a lo largo del tiempo y si la VP es más reducida que el resto, coetánea o vecina. Para ello se ha realizado el levantamiento de la VP que se conserva en Madrid sobre la base de datos pormenorizados del Catastro. La explotación de los resultados da respuesta a las preguntas formuladas, poniendo de manifiesto un empate.

Palabras clave: vivienda social; vivienda mínima; políticas de vivienda; polígonos residenciales; segregación espacial.

\begin{abstract}
The qualitative value of most public housing (VP) in Madrid has been highlighted in several studies, due to the limited resources during their construction. One idea is the VP's lack of size, making it a small dwelling. The article analyzes if Madrid has maintained a particularly small VP between 1940 and 2010. With this information, we present the progression of size during this time period and conclude whether the VP is smaller compared to the rest, coeval or neighbor. We have validated our results by surveying the preserve VP in Madrid, while conforming to a detailed cadaster database. The lecture of results provides answers to the questions above, showing us a draw.
\end{abstract}

Keywords: social housing; minimum housing; housing policies; social neighborhoods; urban segregation.

(*) GI.VS Grupo de Investigación Vivienda Social, Habitabilidad Básica y Patrimonio Urbano. Universidad Politécnica de Madrid (Madrid, España).

Persona de contacto/Corresponding author: c.fsalgado@upm.es (C. Fernández Salgado)

ORCID: http://orcid.org/oooo-0001-9363-0033 (L. Moya González); http://orcid.org/oooo-00o1-7934-6714

(C. Fernández Salgado); http://orcid.org/oooo-0002-6426-3403 (F. Escamilla Valencia)

Cómo citar este artículo/Citation: Moya González, L., Fernández Salgado, C., Escamilla Valencia, F. (2016). Evolución del tamaño de la vivienda de promoción pública y su comparación con el resto del parque residencial construido en Madrid entre 1940-2010. Informes de la Construcción, 69(545): e178, doi: http://dx.doi.org/10.3989/ic.16.040.

Copyright: (C) 2017 CSIC. Licencia / License: Salvo indicación contraria, todos los contenidos de la edición electrónica de Informes de la Construcción se distribuyen bajo una licencia de uso y distribución Creative Commons Attribution License (CC BY) Spain 3.o. 


\section{INTRODUCCIÓN}

El presente artículo analiza la evolución del tamaño de la Vivienda de Promoción Pública (VP) en la ciudad de Madrid, desde el año 1940 y hasta 2010. En esta evolución, además del factor tiempo, se tiene en cuenta el tipo edificatorio y la posición urbana. Ello permite comparar el tamaño de la VP de tres formas: consigo misma a lo largo del tiempo; con la vivienda de promoción no pública (VNP) construida de forma coetánea; así como con la VNP según posición urbana, vecina.

Para poder realizar este análisis, previamente desde el Grupo de Investigación UPM «Vivienda Social» se ha realizado un levantamiento de la VP en Madrid en el período indicado, a partir de las diferentes fuentes parciales publicadas y de la información facilitada por los propios organismos promotores ${ }^{1}$. El levantamiento se ha delimitado sobre la base gráfica y alfanumérica de Catastro ${ }^{2}$. La base catastral se ha trabajado con el objeto de determinar y agrupar la edificación por edades, tipos edificatorios (vivienda colectiva COL y unifamiliar UNI) y clases (distinguiendo la VP del resto del residencial, VNP).

El análisis distingue la Vivienda de Promoción Pública (VP), entendiendo como tal la de promoción exclusivamente directa por parte de la Administración, siendo una categoría restrictiva dentro de los programas de Vivienda Social, incluyendo esta última la Vivienda de Protección. Dentro de la VP existen diferencias según el beneficiario, aunque en este trabajo se considera agrupada. No obstante, cabe distinguir tres grandes grupos en su relación con el tamaño: la VP de inserción social, destinada a un usuario sin recursos; la VP como mecanismo de ayuda a clases medias y medias bajas; y la VP para funcionarios, en ocasiones de un tamaño notablemente mayor y en una posición más céntrica.

En cuanto al período temporal, en este trabajo se identifica la VP construida después de 1940. A partir de esta fecha se articula una política pública de vivienda con continuidad hasta nuestros días. Con anterioridad encontramos que existe una mínima promoción pública municipal al amparo de la legislación de Casas Baratas, que en su conjunto fue más una política de protección de promoción privada (1). No obstante, existen en ese período casos de promoción pública, pertenecientes al antiguo Patronato Municipal de la Vivienda, que fueron adoptados por este organismo después de la Guerra Civil.

El levantamiento de la VP termina en 2010. En este estudio, la VP anterior a 1940 y posterior a 2010 aparece agrupada con la VNP, formando parte del parque construido en esos períodos, sin desglose. El total del parque analizado (VP + VNP) construido en cualquier momento y con registro catastral activo como vivienda en el momento de lectura (enero 2014) son 1.448 .112 viviendas $^{3}$. A ello nos referimos como el $100 \%$ del parque. Como primer dato relevante, el presente estudio identifica que de este total, la VP que se conserva promovida desde 1940 y hasta 2010, supone un 11,1\% (Figura 1).

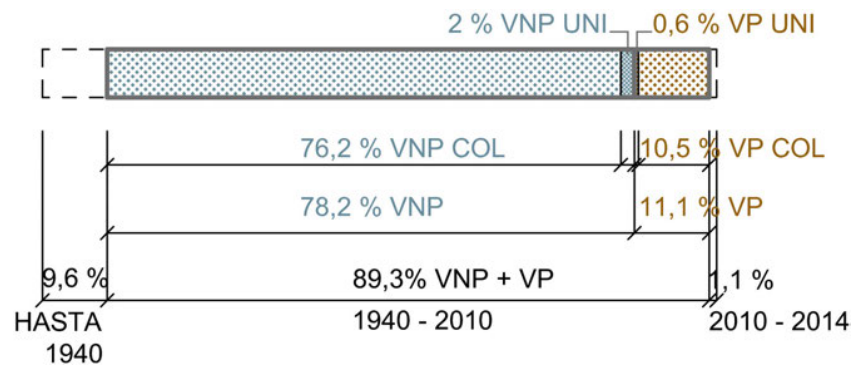

Figura 1. Distribución porcentual del parque residencial madrileño según etapa. En la comprendida entre 1940 y 2010 se distingue entre VNP y VP y, dentro de estos tipos entre COL y UNI. (Fuente: elaboración propia).

\section{PERÍODOS Y CRITERIOS DE ANÁLISIS}

Para caracterizar la historia de la VP en Madrid, entre 1940 y 2010 se han definido cuatro períodos. Estas etapas se acotan por su relación con el contexto político, con el planeamiento regulador, con la intensidad, iniciativa de la promoción y con la mezcla o no de la VP con la VNP en los conjuntos. Diferentes investigaciones determinan el interés por la consideración de períodos quinquenales, en especial por las notables diferencias entre los mismos en la primera etapa (2).

El Período I (P.I), entre 1940 y 1974, abarca toda la VP construida en la Dictadura y se ha dividido en 3 subperíodos (P.I.1, P.I.2 y P.I.3), que pivotan en torno al Plan de Urgencia Social de 1957, que implica notables diferencias. Se trata principalmente de conjuntos de VP en exclusiva en una posición inicial mayoritaria de marcada periferia. El Período II (P.II), entre 1975 y 1989, incluye principalmente el desarrollo del programa Barrios en Remodelación en la Transición, extendido a lo largo de los años ochenta, en una posición excéntrica, pero notablemente menos periférica. El Período III (P.III), entre 1990 y 1999, incluye parte del desarrollo de las previsiones de VP contenidas en el Plan General de 1985, en especial el programa Bolsas de Deterioro Urbano, como sutura urbana de tejidos consolidados, con la singularidad de que su materialización comienza a mezclar la VP/VNP en los conjuntos. El Período IV (P.IV), entre 2000 y 2009, supone el desarrollo mayoritario de los PAUs (Programas de Actuación Urbanística) contenidos como reservas de suelo en el Plan General de 1985, así como los desarrollos previstos en el último Plan General vigente, del año 1997. Este último período se caracteriza por una evolución desde piezas de promoción prioritariamente pública a prioritariamente privada, con mezcla en ambas de VP/VNP en la mayoría de los conjuntos. La posición de las grandes bolsas de suelo de los PAUs se sitúa en el borde urbano tanto Norte como Sur, suponiendo un equilibrio en la promoción de VP en cuanto a posición urbana, que anteriormente se había promovido destacadamente en el Sur.

En cuanto a la intensidad de la promoción, del 11,1\% total de VP, el valor de promoción medio ${ }^{4}$ por quinquenio en cada pe-

\footnotetext{
${ }^{1}$ Se agradece la colaboración en la supervisión del levantamiento de los responsables J. Calatayud (IVIMA) y de M.Á. Prieto (EMVS).

${ }^{2}$ Se agradecen las aclaraciones realizadas por los responsables del Catastro F. de Aragón, L. Arijón, J. Gallego y C. Alonso sobre las principales consideraciones catastrales tenidas en cuenta.

3 El número de viviendas considerado se refiere a la suma de todos los Bienes Inmuebles cuyo uso mayoritariamente es el residencial, obtenido de la base de «Bienes Inmuebles» del Catastro y no de la de «Elementos» (unidades que componen un Bien Inmueble), para el correcto cómputo de superficies en viviendas unifamiliares y dúplex.

4 Dentro de cada período la intensidad de promoción no es constante. En especial destacan los quinquenios Q5, Q7, Q9 y Q12 (Tabla 1).
} 
ríodo (del I al IV) resulta: o,84, 0,78, o,76 y o,67\% (Tabla 1). Se trata de unos valores que implican una promoción de VP progresivamente menor. El dato pone de manifiesto que en los 35 años de Franquismo (Q1-Q7) se construye algo más de VP que en los 35 de Democracia (Q8-Q14), a pesar del desarrollo financiero, organizativo y tecnológico de esta última etapa. El balance sería todavía más favorable en términos de número de promoción para el P.I (1940-1974) al situarse en él la mayor parte de la VP demolida en procesos de remodelación urbana, no tenida en cuenta en este estudio al no ser registros catastrales activos actualmente.

\subsection{La promoción de VP según posición urbana}

Comparada con otras metrópolis europeas - como ya advirtiera Terán (3) - Madrid es una ciudad compacta, en la que una circunferencia con centro en la plaza de Cibeles de sólo $10 \mathrm{~km}$ de radio, abarca el suelo residencial de su término municipal. Este criterio de distancia se ha mantenido hasta nuestros días, si evitamos la consideración del suelo residencial de los municipios limítrofes, que participan en la conformación de su Área Metropolitana.

Para determinar un criterio de posición urbana, en continuación con el utilizado en la investigación sobre barrios de VP hasta la dictadura realizada por Moya (4), se han definido tres coronas concéntricas con centro en la plaza de Cibeles y radios $3 \mathrm{~km}$., $6 \mathrm{~km}$ y una última hasta el límite del término municipal ${ }^{5}$. La segunda corona, entre los 3-6 km, conforma una primera periferia en la que se sitúa el grueso de la VP. Esta distancia (3 km) fue elegida desde los primeros estudios por ser el límite aproximado del Madrid de después de la Guerra Civil y el ancho elegido - también aproximado- para clasificarlo como Green Belt en el Plan General de 1946.

El crecimiento de Madrid resulta parcialmente radioconcéntrico, al excluir la posición Noroeste ocupada por la Casa de Campo y el Monte del Pardo. En cuanto a la sectorización, se apoya en las vías radiales de conexión de la capital con el territorio. El paseo de la Castellana marca un nítido eje NorteSur. La sectorización la componen vías de alta intensidad y barreras urbanas como el ferrocarril.

Para poder matizar las posibles diferencias de superficie en el parque residencial, en este estudio se ha considerado la división del total del término municipal de Madrid en 21 sectores-corona, establecidos en relación con la historia del desarrollo urbano y de las infraestructuras descritas (Figura 2 y Figura 4.4). Esta consideración elude voluntariamente la división en barrios administrativos, por ser de un tamaño excesivo en la corona periférica, sin responder además a la formalización de una identidad morfológica y funcional de referencia ${ }^{6}$.

En cuanto a la consideración de un Norte -más rico- y un Sur -más pobre- en la ciudad de Madrid, ésta es recurrente en los estudios sobre rentas urbanas y segregación espacial, sin una delimitación espacial concreta y coincidente (5), (6). En Madrid, el Norte ha tenido un mayor atractivo urbano por su mayor altitud, calidad del terreno y relación con el paisaje natural. En este estudio se ha considerado como frontera entre el Arco Norte (ARC NOR) y el Arco Sur (ARC SUR) la línea que discurre desde la carretera de Barcelona, atravesando la ciudad por la calle Alcalá y continuando por la de Extremadura (Figura 4.4). En coherencia con la teoría de la posición urbana de las rentas más desfavorecidas descrita por Secchi (7), el estudio pone de manifiesto cómo la VP se ubica mayoritariamente en el ARC SUR, donde se identifica que se posiciona el 8,8\% del 11,1\% total (Tabla 1 y Figura 3).

\subsection{Edades de la edificación y tipos}

A partir de la identificación de las edades de la edificación?, el estudio permite observar el enorme peso que en Madrid tiene la periferia reciente - de «aluvión»- al constatar que entre 1940 y hasta 2010 se promueve el 89,3\% del parque existente actualmente (VP + VNP). Únicamente el 9,6\% del parque vivienda madrileño (VP + VNP) es anterior a 1940 en todo el término municipal, localizándose preferentemente en el centro histórico y en los cascos históricos anexionados.

En cuanto al tipo edificatorio, el período analizado coincide en la historia del urbanismo con el predominio de las variaciones de tipos abiertos - principalmente bloque lineal y torre- promovidos fundamentalmente de forma unitaria mediante la lógica de crecimiento urbano de los polígonos. En la VP madrileña se dan casos de promoción entre medianeras, representando, sin embargo, un porcentaje minoritario. No obstante, por las restricciones de este estudio, únicamente se distingue entre las categorías de vivienda unifamiliar UNI y colectiva COL, cuya consideración tiene una importante repercusión con respecto al tamaño de los respectivos parques.

En origen, como continuación de la vivienda protegida anterior a la Guerra Civil, debido a las carencias en los modos y materiales de construcción, se promovió un amplio porcentaje de VP unifamiliar en hilera (Tabla 1), de sencilla ejecución. Pero en conjunto, con respecto al 11,1\%, el estudio revela una enorme diferencia entre el uso la $\operatorname{COL}(10,5 \%)$ frente a la UNI (o,6\%). Igualmente, con respecto al parque total (VP + VNP) construido entre 1940-2010 (89,3\%), la COL supone mayoritariamente el $86,7 \%$ frente a un 2,6\% UNI. Es por ello que en el conjunto de los análisis que se realizan a continuación se prioriza la comparación de la COL, por suponer la gran mayor parte del parque.

\subsection{Rangos de tamaño}

Sobre la consideración de si la VP es pequeña o grande, cabe señalar que, comparada con el techo de tamaño del parque residencial de Madrid, la VP será siempre pequeña. Pero sin

\footnotetext{
5 En el caso de que las coronas 1 y 2 intersequen conjuntos con VP, se han deformado para incorporarlos enteros en aquella corona en la que tienen una mayor superficie.

${ }^{6}$ Como ejemplo, el barrio de Buenavista, que incluye tanto el Casco Histórico de Carabanchel como su gran PAU o ensanche; o el barrio de Casco Histórico de Vallecas, que, además del mismo, incluye igualmente la enorme pieza urbana de su PAU o ensanche reciente. Ambos PAUs tienen importante promoción de VP.

7 En este estudio la edad de la edificación refleja la categoría «registros de construcción» de la base catastral. En caso de que la edificación haya sufrido una ampliación o reforma integral, el Catastro indica la edad de la reforma. En estos casos se ha mantenido la consideración catastral, por entender, como lo hace el Catastro, que supone una nueva realidad patrimonial.
} 


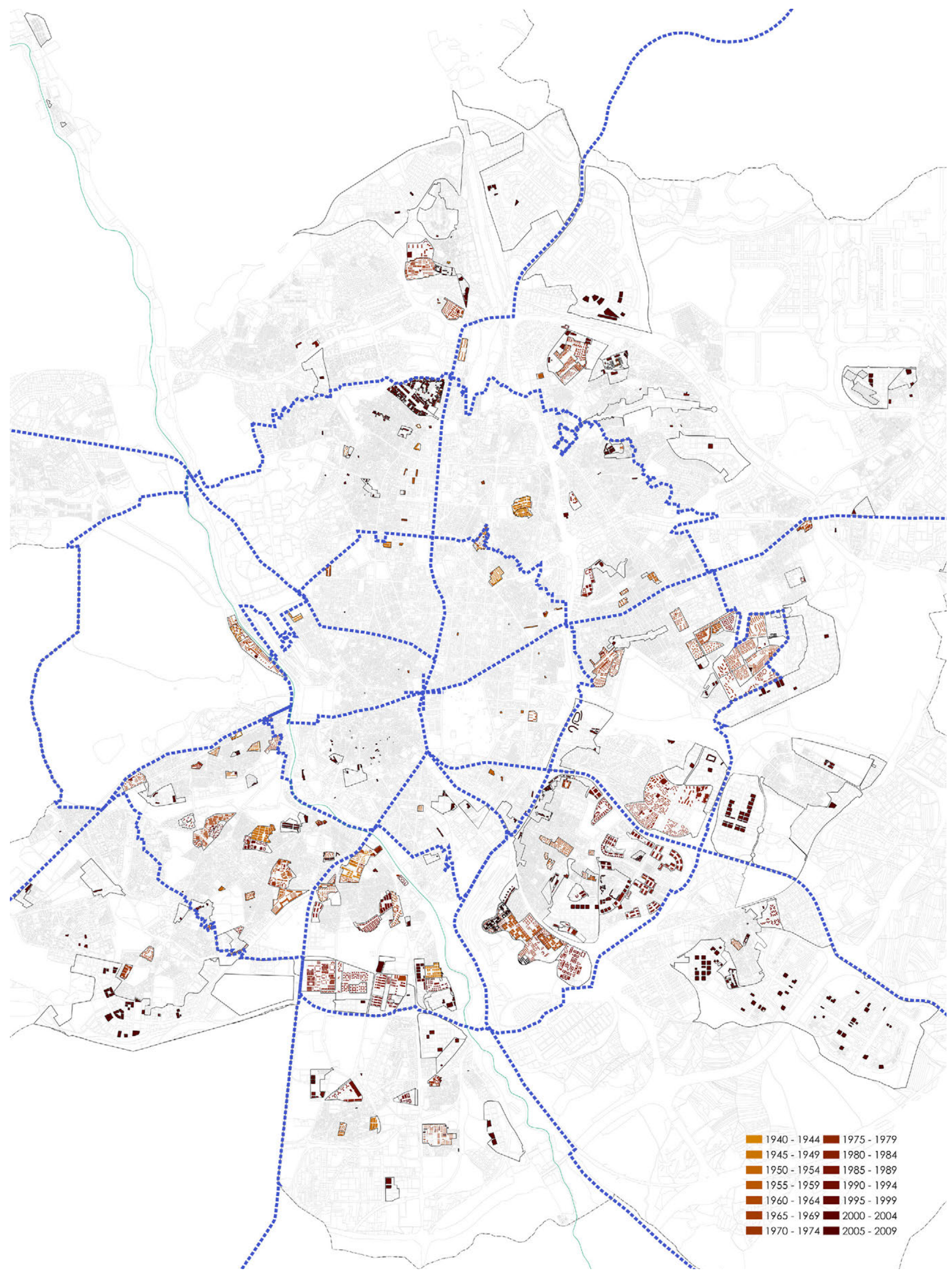

Figura 2. Localización de la VP en el término municipal de Madrid sobre el parcelario catastral. El color representa el quinquenio de promoción según leyenda. Las líneas azules representan la división en sectores-corona propuesta. Las coronas se corresponden con una distancia de 3 y $6 \mathrm{~km}$ tomando como centro la plaza de Cibeles, deformadas para incluir enteras aquellas piezas urbanas de promoción unitaria en la que tengan mayor superficie. En línea negra se representan el límite de conjuntos de promoción unitaria con VP. Las parcelas coloreadas que no están incluidas dentro de un conjunto son promociones aisladas de VP. (Fuente: elaboración propia). 


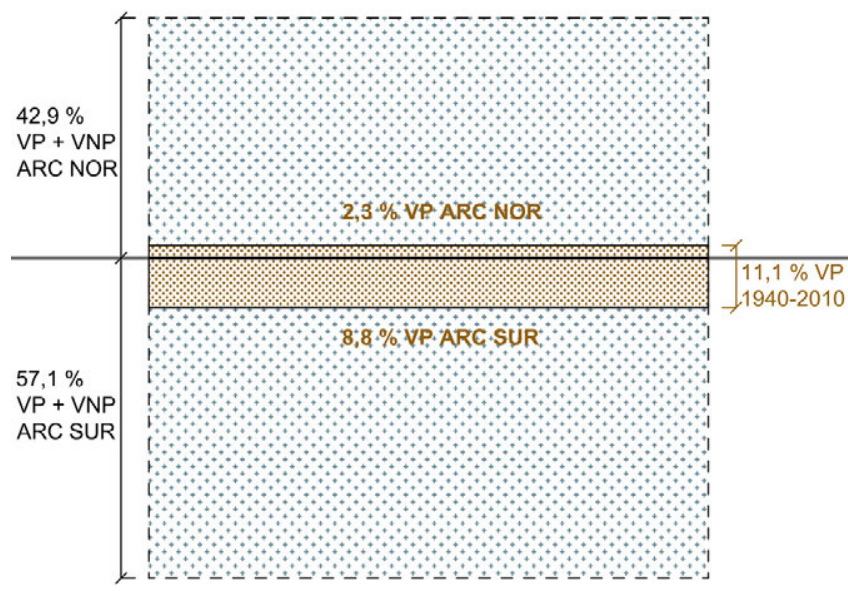

Figura 3. Distribución porcentual del parque residencial en Madrid según posición urbana (Arcos Norte y Sur). Se diferencia el peso de la VP promovida entre 1940 y hasta 2010. (Fuente: elaboración propia).

serlo en un valor absoluto, lo que este estudio pretende es analizar comparativamente el tamaño mediante una distribución porcentual según rangos de superficie definidos ${ }^{8}$. En base a la definición de esos rangos es posible analizar en qué posición se sitúa el tamaño del parque de VP. En la determinación de los valores de los rangos se ha tenido en cuenta trabajos realizados anteriormente (8).

El dato tomado del tamaño de la vivienda se define como «superficie construida privativa computable a efectos de Catastro» ${ }^{9}$. Por ser de uso más común las superficies útiles y de mayor interés para este estudio, al relacionarse con condiciones de habitabilidad, el anterior dato se ha transformado a una superficie útil privativa $\left(\mathrm{m}^{2} \mathrm{u}\right)$, estableciendo un factor de conversión medio de 1,15 como resultado de dividir la anterior superficie construida privativa computable $\left(\mathrm{m}^{2} \mathrm{c}\right)$ entre la útil. De esta forma se ha establecido la siguiente distribución en 5 rangos: $\mathrm{R} 1\left(<51,75 \mathrm{~m}^{2} \mathrm{c} »<45 \mathrm{~m}^{2} \mathrm{u}\right)$; R2 (51,75-69 $\left.\mathrm{m}^{2} \mathrm{c} » 45-60 \mathrm{~m}^{2} \mathrm{u}\right) ; \mathrm{R} 3\left(69-86,5 \mathrm{~m}^{2} \mathrm{c} \gg 60-75 \mathrm{~m}^{2} \mathrm{u}\right) ; \mathrm{R} 4$ (86,5$\left.103,5 \mathrm{~m}^{2} \mathrm{c} » 75-90 \mathrm{~m}^{2} \mathrm{u}\right) ; \mathrm{R}_{5}\left(>103,5 \mathrm{~m}^{2} \mathrm{c} »>90 \mathrm{~m}^{2} \mathrm{u}\right)$.

Para poder establecer una comparación se ha traducido el número de viviendas de cada horquilla de tamaño a porcentaje sobre el total de cada categoría. Es decir, entre los rangos 1 y 5 suman siempre el $100 \%$. La distribución en 5 rangos permite diferenciar un rango central $\mathrm{R}_{3}$, flanqueado por dos pequeños ( $\mathrm{R} 1$ y R2) y dos grandes ( $\mathrm{R} 4$ y $\mathrm{R} 5$ ). El criterio utilizado es que el parque es grande en la suma de rangos $\mathrm{R} 4$ + R5 y pequeño en la suma R1 + R2. La comparación para determinar que una categoría es más grande que otra se establece en base a una doble condición: tener mayor porcentaje de viviendas en los rangos grandes $\left(\mathrm{R}_{4}+\mathrm{R} 5\right)$, pero también menor porcentaje en los pequeños ( $\mathrm{R} 1+\mathrm{R} 2)$.
Podría parecer a priori - en la idea de que la VNP en Madrid es grande-, que el $\mathrm{R}_{5}$, que recoge la vivienda que supera los $90 \mathrm{~m}^{2} \mathrm{u}$, tendría un alto valor. Ello implicaría un bajo conocimiento de los datos desagregados. Sin embargo, la medición real de los datos que arroja este estudio pone de manifiesto que el $81,6 \%$ del total del parque tiene una superficie inferior a $90 \mathrm{~m}^{2} \mathrm{u}$. Ello sirve como verificación previa de los rangos adoptados, que para el $100 \%$ del parque (VP + VNP) es: R1 (15,37\%), R2 (24,56\%), R3 (25,33\%), R4 (16,35\%) y R5 $(18,39 \%)$ (Tabla 2$)$. La vivienda en posiciones centrales de Madrid puede alcanzar superficies grandes, pero al mismo tiempo existen viviendas pequeñas, incluso en algunos casos infraviviendas ${ }^{10}$.

\section{EL TAMAÑO DE LA VP EN MADRID}

En la línea de la vivienda social europea, construida de una forma masiva tras la Segunda Guerra Mundial, en España la primera VP es una vivienda de dimensiones reducidas. Así algunos proyectistas de barrios de VP recuerdan cómo la Administración reducía recursos y aumentaba los programas durante su elaboración, para construir más viviendas ${ }^{11}$. A medida que el país abandonaba la depresión económica producida tras su Guerra Civil, la política social de vivienda se iría adaptando a unas dimensiones mayores, llegando en la vivienda protegida a tamaños considerables (9), (10).

En el caso de la VP de Madrid el gran salto de calidades, tamaño y mejora en la posición urbana se producirá en la Transición y Primera Democracia, entre finales de los años setenta y a lo largo de los ochenta. En ese momento la presión social de los habitantes de antiguos barrios de VP degradados logra su remodelación, cuestionando las calidades y tamaños de esa vivienda mínima. El resultado fue el salto cualitativo y cuantitativo que supone el programa de la Operación de Remodelación y Realojamiento de Barrios en Madrid (11). Tras ello, progresivamente la VP pasará de promoverse por conjuntos a promoverse de forma puntual dentro de conjuntos de promoción privada. En cuanto a proyecto y paisaje urbano ello supone el paso de conjuntos «comparsa» de VP a una VP aislada, con vocación de «hito» (12). En lo referente a su tamaño, éste irá disminuyendo, en sintonía con el de la VNP en los nuevos desarrollos, como ponen de manifiesto los datos de esta investigación.

El estudio revela que no existen notables diferencias en cuanto a la comparación del tamaño de la VP en las posiciones Norte (ARC NOR) y Sur (ARC SUR). Tomando como referencia la mayoritaria categoría COL, los datos reflejan (Tabla 3) que a partir de una situación de empate en el R3 en torno al $30 \%$, los rangos altos $\left(\mathrm{R}_{4}+\mathrm{R}_{5}\right)$ suman en ambos arcos algo más de un $33 \%$ y existe una diferencia de menos de un punto en la suma de rangos bajos (R1 + R2). Por tanto, en cuanto

\footnotetext{
${ }^{8}$ Se ha descartado la utilización de la media o la mediana, por no reflejar los valores extremos, que se consideran esenciales en este estudio.

9 Atendiendo a la definición catastral: «Se entiende como superficie construida la superficie incluida dentro de la línea exterior de los parámetros perimetrales de una edificación y, en su caso, de los ejes de las medianerías, deducida la superficie de los patios de luces. Los balcones, terrazas, porches y demás elementos análogos, que estén cubiertos, se computarán al 50 por 100 de su superficie, salvo que estén cerrados por tres de sus cuatro orientaciones, en cuyo caso se computarán al 100 por 100. En uso residencial no se computarán como superficie construida los espacios de altura inferior a 1,50 metros». Fuente: Norma 11.3 del Real Decreto 1020/1993.

${ }^{10}$ Detectado, entre otros, en el Estudio y Propuesta Normativa de las condiciones básicas del uso residencial para la Revisión del Plan General de Madrid. L. Moya [Dir.]. C. F. Salgado y E. L. de Munaín. GI.VS, UPM, 2011 (no publicado), basado en datos suministrados por el Ayuntamiento y otros estudios sobre la materia.

${ }^{11}$ Romany, arquitecto autor de, entre otros proyectos, el Poblado Dirigido de Fuencarral en Madrid, ponía de manifiesto esta circunstancia en una entrevista en enero de 2015 con L. Moya.
} 
a tamaño, podemos considerar un equilibrio en la VP entre ambas posiciones urbanas, no obstante marcadamente diferentes - como se ha señalado anteriormente- en cuanto a intensidad de promoción (Figura 3).

\subsection{Evolución del tamaño de la VP por período}

Analizando la evolución del tamaño de la VP a lo largo del tiempo, se observa cómo en el P.I.1 (1940-1954) la VP COL es pequeña, pero lo es todavía más en el P.I.2 (1955-1964), que tiene un importantísimo peso por suponer el desarrollo del Plan de Urgencia Social, fundamentalmente en el Q5. En el P.I.3 (1965-1974) la VP COL crece ligeramente sobre su propia media, pero no será hasta el P.II, que coincide como se ha señalado con el programa Barrios en Remodelación, cuando el parque de VP COL alcance el mayor de los tamaños. A partir de ese momento el parque de VP COL seguirá siendo superior a su media global, pero reduciendo su tamaño en el P.III y aún más en el P.IV. En detalle los resultados del análisis son (Tabla 4 y Figura 4.1):

En el P.I (5,86\% del $100 \%$ total del parque residencial existente) la promoción de VP destaca en el ARC SUR COL (4,3\%). Comparada esta categoría con la VP TOT COL hay muchas menos viviendas grandes ( $<\mathrm{R} 4$ y $\left.\mathrm{R}_{5}\right)$, baja también el porcentaje del rango medio $\left(<\mathrm{R}_{3}\right)$ y hay más viviendas pequeñas ( $>$ R1 y R2). Por tanto, con respecto a su media, la VP en el P.I es mucho más pequeña.

En el P.II (2,35\% del total) la promoción de VP destaca en el ARC SUR COL ( $2 \%$ ). Tomando como referencia esta categoría, con respecto al P.I, en cuanto a la distribución de rangos, se reduce drásticamente el R1, desplazando el mayor peso al R4 (56,6\%). El R5 es notablemente mayor (15,2\%). Los rangos 4 y 5 son el doble de la media total de VP y los rangos R1 y R2 son mucho menores. Por tanto, en el P.II se trata de una vivienda mucho más grande que en el período anterior y que la media de VP.

En el P.III (1,52\% del total) la promoción de VP destaca igualmente en la posición ARC SUR COL (1,13\%). Con respecto al P.II, en cuanto a la distribución de rangos, el grueso sigue llevándolo el R4 (43\%), aunque disminuye desde un $56 \%$ anterior, triplicándose los rangos $\mathrm{R} 2$ y R3 con respecto al P.II. En conjunto en este período hay un ligero desplazamiento hacia rangos de tamaño inferiores con respecto al período anterior, siendo no obstante superior en tamaño al del conjunto de la VP.

En el P.IV (1,33\% del total) la promoción de VP se centra de nuevo en ARC SUR COL (o,9\%). Con respecto al período anterior, en cuanto a la distribución de rangos, la promoción no varía en el $\mathrm{R}_{3}$ (en torno al $32 \%$ ). Los rangos superiores $\mathrm{R} 4$ y $\mathrm{R}_{5}$ disminuyen notablemente y en paralelo aumentan en gran medida los inferiores, R1 y R2. En el P.IV se trata, por tanto, de un parque de VP de tamaño inferior con respecto al período anterior, pero ligeramente superior al de la media de VP.

\subsection{Comparación VP/VNP coetánea}

Si en el período analizado comparamos el tamaño de la VP COL (mayoritaria, con un 10,5\%) con el total de la VNP COL (igualmente mayoritaria, con un 76,2\%) (Tabla 2), resulta que hay más porcentaje de VNP de las más grandes (> R5),

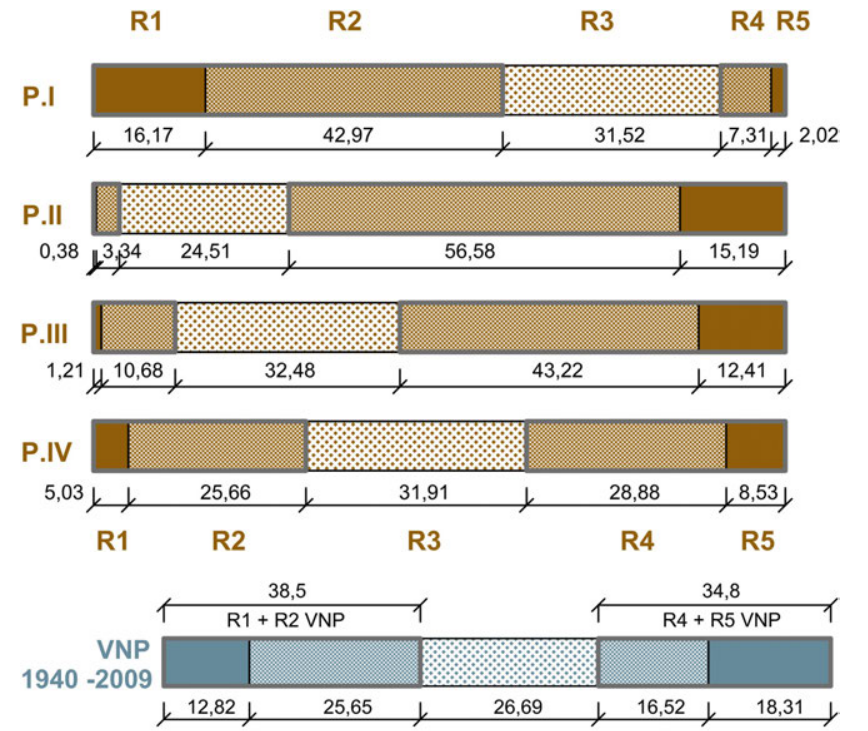

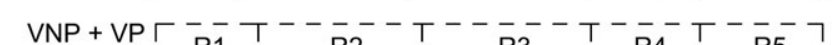
hasta $2014 \mathrm{R} 14 \mathrm{R} 2 \quad \mathrm{R} 3 \quad \mathrm{R} 4 \mathrm{R} 5$
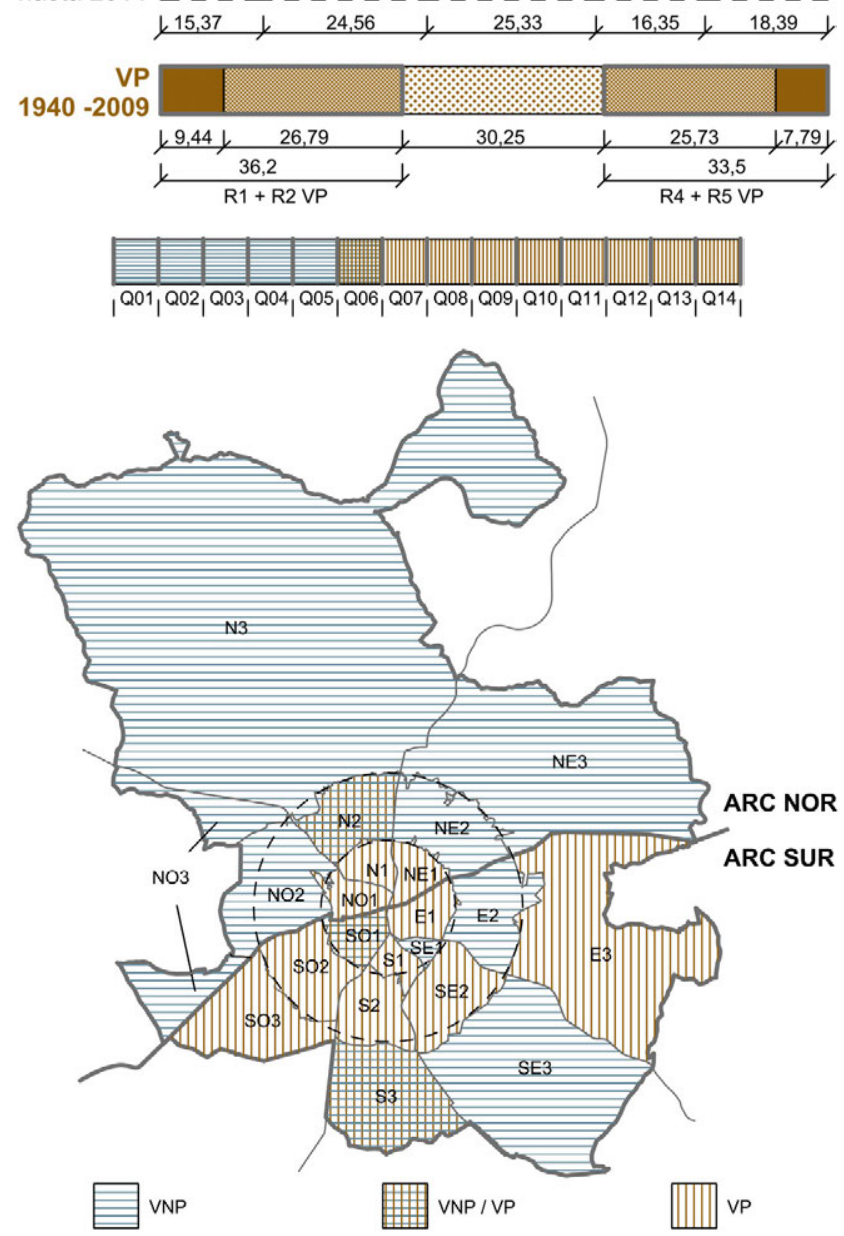

Figura 4. [4.1] Distribución porcentual del tamaño de la VP en la categoría COL y posición ARC SUR según período (I-IV). Se destacan más intensamente los rangos bajos (R1, R2) y, en el otro extremo, los rangos altos (R4, R5); [4.2] Distribución de los rangos de tamaño de la VNP (en azul) y de la VP (en marrón) en la categoría COL, mayoritaria, en el período 1940-2010; [4.3] Predominio del tamaño del parque de VNP (azul)/VP (marrón) según quinquenio a partir de los datos desglosados del estudio, en base a la comparación de la mayoritaria categoría de vivienda COL en la posición ARC SUR; [4.4] Predominio del tamaño del parque de VNP (azul)/VP (marrón), o mezcla de ambos en situación de empate, según posición urbana en los Arcos Norte y Sur, Coronas y Sectores, comparando la categoría mayoritaria (COL promovida en el período 1940-2010). (Fuente: elaboración propia). 
pero también más porcentaje de VNP de las más pequeñas ( $>\mathrm{R} 1)$. La suma de los rangos mayores $\left(\mathrm{R}_{4}+\mathrm{R} 5\right)$ es similar en ambas, siendo un $34,8 \%$ en VNP y un $33,5 \%$ en VP. La pequeña diferencia entre ambas, favorable en este caso a la VNP, es igual a la diferencia, favorable a la VP en los rangos inferiores. La suma de éstos (R1 + R2) es 38,5\% en el caso de la VNP y de $36,2 \%$ en el caso de la VP. Por tanto, teniendo en cuenta todo ello, podemos considerar que el parque de VNP y de VP es de similar tamaño en la mayoritaria categoría COL, pudiendo hablar de un empate en cuanto a tamaño del parque (Figura 4.2).

En cuanto a la comparación de la VP con la VNP coetánea, por períodos, en la categoría mayoritaria (COL en el ARC SUR), se produce la misma lógica que la de la evolución de la VP consigo misma: la VP es más pequeña que la VNP en el P.I, aunque al final de este período la situación se invierte. En el P.II la VP es notablemente más grande que la VNP y en los P.III y IV sigue siendo algo más grande que la VNP, pero sin tanta diferencia como en el P.II. (Figura 4.3).

\subsection{Comparación VP/VNP colindante}

Si comparamos la VP con la VNP distinguiendo las posiciones ARC NOR y SUR (Tabla 3), en la categoría mayoritaria (COL promovida entre 1940-2010), la VNP es mayor que la VP en el ARC NOR, al tener notablemente más R5 (27,7\% frente a 9,7\% en VP) y ser la suma de rangos altos ( $\mathrm{R}_{4}+\mathrm{R}_{5}$ ) favorable a la VNP (43,8\% frente a un $33,2 \%$ en VP). Aunque la VNP tiene más R1 (14,7\% frente a 10,5\% de la VP) la suma de rangos bajos (R1 + R2) es igual en ambas (en torno al 35\%).

En el ARC SUR, donde la VP COL es mayoritaria por suponer un $8,3 \%$ sobre el $11,1 \%$ total, la situación es inversa. La VNP tiene ligeramente más $\mathrm{R}_{5}$ ( $11 \%$ frente a $7,3 \%$ de la VP), pero la suma de rangos altos ( $\left.\mathrm{R}_{4}+\mathrm{R}_{5}\right)$ es favorable a la VP, que suma $33,6 \%$ frente a los $27,8 \%$ de la VNP. Además en VP el R1 y R2 son ambos menores, resultando su suma 36,4\% frente al $41 \%$ en VNP.

La comparación VP/VNP teniendo en cuenta la Corona (COR $1,2,3), \operatorname{los}$ Arcos (ARC NOR y SUR) y los sectores (NO, N, $\mathrm{NE}, \mathrm{E}, \mathrm{SE}, \mathrm{S}, \mathrm{SO}$ ), tomando como referencia los valores de la categoría notablemente mayoritaria (COL promovida entre 1940-2010), arroja los siguientes resultados (Tabla 5 y Figura 4.4) en el ARC NOR: en la COR1, la VP es mayor que la VNP en los tres sectores ${ }^{12}$; en la COR 2 la situación es inversa, favorable a la VNP, a excepción del N2 -que se correspondería con la zona de Tetuán- donde el tamaño es similar; la COR 3 se decanta por una VNP mayor en tamaño que la VP.

El ARC SUR, donde como hemos señalado se ha promovido mayoritariamente la VP, ésta es mayor en tamaño en la COR1, claramente así en las posiciones E1 y S1; en el SO1 hay un equilibrio de tamaños y en el SE 1 es mayor la VNP; en la COR2, la situación global es favorable a la VP, siéndolo así en tres de los cuatro sectores, mientras que en el E2 la situación es favorable a la VNP; en la COR3 las variaciones vuelven a ser notables, predominando el tamaño de la VP en el $\mathrm{E}_{3}$ y $\mathrm{SO}_{3}$, mientras que en el $\mathrm{S}_{3}$ los tamaños son equilibrados y en el SE3 resulta un tamaño mayor el de la VNP en comparación con el de la VP.

\section{CONCLUSIONES}

De los resultados de este estudio podemos destacar que el parque residencial de Madrid se construye principalmente entre 1940 y 2010, al promoverse el 89,3\% de lo actualmente existente. Ello manifiesta el fortísimo peso de la periferia sobre el total del término municipal. Es en ella, en un limitado radio - principalmente entre los 3-6 km- en donde se ha construido la VP. Ésta supone el 11,1\% del total existente. El paseo de Extremadura, al Oeste, y la calle Alcalá, al Este, permiten una delimitación Norte/Sur. Es en ese Sur en el que se ha promovido principalmente la VP $(8,8 \%)$.

Del 11,1\% total de VP el 5,9\% se corresponde con el franquismo, frente a un 5,2\% del período democrático, en el que se observa cómo la media de promoción es progresivamente decreciente. La construcción de VP destaca en cuatro momentos: el impacto del Plan de Urgencia Social (Q5); un repunte de la promoción pública en la última dictadura (Q7), que se correspondería con barrios como Moratalaz; la implementación del programa Barrios en Remodelación (Q9-Q10); y su continuidad con una menor intensidad mediante el programa Bolsas de Deterioro Urbano (Q11-Q12).

En cuanto a la evolución de la superficie de la VP y su comparación con la de la VNP coetánea, la primera comenzó siendo una vivienda notablemente reducida, con un desfase de tamaño desfavorable con respecto a la segunda. Es en la Transición - Primera Democracia (P.II) cuando se produce un salto de tamaño de la VP respecto de su propia media. Dicho período supone además que la VP adelanta notablemente en tamaño a la VNP. Tras ello, la VP redujo su superficie y de forma paralela también lo hizo el parque de VNP, en los Períodos III y IV. En ellos, no obstante, el tamaño de la VP se ha mantenido ligeramente por encima del de la VNP coetánea, aunque sin una diferencia tan notable como en el P.II.

Los matices en cuanto a la comparación del tamaño del parque de VP/VNP, según posición urbana, ponen de manifiesto situaciones dispares, pero al mismo tiempo una idea clara: el tamaño de la VP madrileña no dista del de la VNP vecina; en ocasiones lo supera y en otras se queda por detrás. En conjunto, para todo el término municipal, se trata de un tamaño tan parecido que, en una consideración agrupada, supone lo que podríamos definir como «empate». Por tanto, las características de tamaño de la VP en Madrid no debieran implicar un factor estigmatizante, como parque especialmente pequeño.

A partir de estos datos de base se abren diferentes vías de estudio. En especial si la variación del tamaño de la VP se ha acompasado con la variación de las estructuras familiares. Sobre ello se parte de la hipótesis de que no es así1 ${ }^{13}$, tratándo-

\footnotetext{
${ }_{12}$ El dato se explicaría teniendo en cuenta que en esta posición (VP COL + UNI COR 1 1940-2010) la intensidad de promoción de VP es reducida (o,2\% del 11,1\% del total), siendo en muchos casos viviendas para el colectivo de funcionarios, de mayor tamaño como se ha señalado inicialmente, y por existir numeroso parque VNP de reducido tamaño -incluso infravivienda - entremezclada con la gran vivienda burguesa. ${ }^{13}$ El estudio de L. Moya y J. Vinuesa de 1986 sobre la operación Barrios en Remodelación (no publicado) explota el detallado censo de ocupantes realizado en 1985 por el IVIMA, poniendo de manifiesto cómo en los barrios de VP remodelados -en los que aumentaba la superficie de vivienda - el número de ocupantes por vivienda era menor que los originales, al tratarse de una población envejecida con hijos emancipados.
} 
se más bien de un ajuste a los estándares del mercado libre. Otra línea de estudio supondría continuar la reflexión sobre el tamaño, características y flexibilidad de los programas de VP, para optimizar los recursos públicos y dar cobertura a un mayor número de usuarios. Ello no implicará únicamente la construcción de una vivienda especialmente reducida, aunque ésta sería protagonista, habida cuenta del reducido tamaño de un gran porcentaje de los actuales hogares.

Una tercera línea podría derivar en el estudio socioeconómico del sostenimiento de un porcentaje de promoción pública como patrimonio social reciclable. Para ello, como señala Vinuesa (13), se debería fomentar la diversidad en la tenencia del parque, en especial incrementando el porcentaje en alquiler. El uso de Sistemas de Información Geográfica (GIS), como el utilizado en este estudio, ayudaría en su gestión ${ }^{14}$ y posibilitaría el cruce con otros datos existentes de carácter físico y socioeconómico, de cara a un continuo mantenimiento de la periferia social en su absorción como parte inherente de la ciudad.

Además, al presente conocimiento del parque de vivienda de promoción pública en sus diferentes regímenes de tenencia, se podrían unir estudios similares sobre el parque de la vi- vienda protegida. El conocimiento del volumen y características del conjunto de estos parques serviría para fijar posiciones de estrategia urbana en cuanto a políticas de vivienda social, superando la regulación urbanística española, que únicamente controla los porcentajes de vivienda no libre en nuevas transformaciones urbanísticas. Su discusión como modelo podría ser similar a la fijación de unos porcentajes de Vivienda Social ${ }^{15}$ en el urbanismo francés, en el que se pretende cubrir un 20-25\% del parque en un horizonte temporal próximo ${ }^{16}$. En el estudio de su localización se habrá de evitar la conformación de los guetos que suponen algunas banlieus europeas (14) y corregir sus desequilibrios, teniendo especialmente en cuenta su potencial vulnerabilidad (15). En su discusión tendrá un mayor sentido ampliar el ámbito al área metropolitana de una gran ciudad como Madrid y no estrictamente al límite de su término municipal.

La puesta en práctica de un modelo urbano cohesivo, en el que esté presente tanto una promoción pública sostenida en el tiempo como su mantenimiento, debiera ser una meta en el marco de mejora de un sistema del bienestar avanzado. En este sentido, Secchi (7) nos recuerda cómo las brechas urbanas han crecido especialmente en contextos desarrollados, ahondando en la conformación de un urbanismo para ricos y pobres.

\footnotetext{
${ }^{14}$ Como ejemplo, destaca el visor GIS que utilizan organismos en materia de VP en régimen de alquiler como la New York City Housing Authority, NYCHA. www.nyc.gov/nycha.

${ }^{15}$ La Vivienda Social en Francia se entiende referida mayoritariamente al parque de alquiler social, tanto de promoción pública como privada, mediante sociedades sin ánimo de lucro, siendo marcadamente diferente en este sentido del caso español, en el que predomina la propiedad.

${ }^{16}$ El porcentaje inicial de Vivienda Social se fija en la Ley de solidaridad y renovación urbana de 13 de diciembre de 200o, conocida como Ley SRU. No obstante, recientes estudios, como los del diario Le Monde (2015.03.19), ponen de manifiesto cómo todavía en muchos municipios no se han alcanzado los objetivos de la Ley en los plazos previstos.
} 
ANEJO

Tabla 1. Peso VP por quinquenio.

\begin{tabular}{|c|c|c|c|c|c|c|c|c|c|c|c|c|c|c|c|c|}
\hline \multirow{2}{*}{ POSICIÓN } & \multirow{2}{*}{ TIPO } & \multicolumn{3}{|c|}{$\begin{array}{c}\text { P.I.1 } \\
\text { 1940-1954 }\end{array}$} & \multicolumn{2}{|c|}{$\begin{array}{c}\text { P.I.2 } \\
\text { 1955-1964 }\end{array}$} & \multicolumn{2}{|c|}{$\begin{array}{c}\text { P.I.3 } \\
\text { 1965-1974 }\end{array}$} & \multicolumn{3}{|c|}{$\begin{array}{c}\text { P.II } \\
1975-1989\end{array}$} & \multicolumn{2}{|c|}{$\begin{array}{c}\text { P.III } \\
\text { 1990-1999 }\end{array}$} & \multicolumn{2}{|c|}{$\begin{array}{c}\text { P.IV } \\
\text { 2000-2009 } \\
\end{array}$} & \multirow{2}{*}{\begin{tabular}{|c|} 
TOTAL \\
VP \\
1940- \\
2009
\end{tabular}} \\
\hline & & \begin{tabular}{|c|} 
Q1 \\
40-44 \\
\end{tabular} & \begin{tabular}{|c|} 
Q2 \\
$45-49$ \\
\end{tabular} & \begin{tabular}{|c|} 
Q3 \\
50-54 \\
\end{tabular} & $\begin{array}{c}\text { Q4 } \\
55-59\end{array}$ & $\begin{array}{c}\text { Q5 } \\
60-64\end{array}$ & \begin{tabular}{|c|} 
Q6 \\
65-69 \\
\end{tabular} & $\begin{array}{c}\text { Q7 } \\
70-74 \\
\end{array}$ & \begin{tabular}{|c|} 
Q8 \\
$75-79$ \\
\end{tabular} & \begin{tabular}{|c|} 
Q9 \\
$80-84$ \\
\end{tabular} & \begin{tabular}{|c|} 
Q10 \\
85-89
\end{tabular} & \begin{tabular}{|c|} 
Q11 \\
$90-94$ \\
\end{tabular} & \begin{tabular}{|c|} 
Q12 \\
95-99 \\
\end{tabular} & \begin{tabular}{|c|} 
Q13 \\
oo-04 \\
\end{tabular} & \begin{tabular}{|c|} 
Q14 \\
05-09
\end{tabular} & \\
\hline \multirow{2}{*}{ ARC NOR } & VP COL & 0,09 & 0,05 & 0,13 & 0,14 & 0,36 & 0,24 & 0,13 & 0,07 & 0,11 & 0,08 & 0,14 & 0,18 & 0,21 & 0,21 & 2,14 \\
\hline & VP UNI & 0,04 & o & 0,01 & o & 0,03 & 0,03 & o & o & o & o & o & 0,01 & 0,01 & o & 0,15 \\
\hline \multirow{2}{*}{ ARC SUR } & VP COL & 0,12 & 0,06 & 0,14 & 0,56 & 1,88 & 0,29 & 1,26 & 0,3 & 1,05 & 0,65 & 0,48 & 0,65 & 0,34 & 0,55 & 8,34 \\
\hline & VP UNI & 0,02 & 0,02 & 0,03 & 0,13 & 0,08 & 0,01 & o & 0,02 & 0,06 & 0,01 & 0,02 & 0,04 & o & 0,01 & 0,45 \\
\hline $\begin{array}{l}\text { ARC NOR + } \\
\text { SUR }\end{array}$ & $\begin{array}{r}\mathrm{VP} \\
\mathrm{COL}+\mathrm{UNI} \\
\end{array}$ & 0,27 & 0,13 & 0,32 & 0,83 & 2,36 & 0,56 & 1,39 & 0,39 & 1,21 & 0,75 & 0,64 & 0,88 & 0,57 & 0,77 & 11,1 \\
\hline \multicolumn{2}{|c|}{ MEDIA POR PERÍODO } & \multicolumn{7}{|c|}{0,84} & & 0,78 & & \multicolumn{2}{|c|}{0,76} & \multicolumn{2}{|c|}{0,67} & - \\
\hline \multicolumn{2}{|c|}{ DICTADURA/DEMOCRACIA } & \multicolumn{7}{|c|}{5,9} & \multicolumn{7}{|c|}{5,2} & 11,1 \\
\hline
\end{tabular}

Peso en \% de la promoción de VP en Madrid sobre el total (VP + VNP hasta 2014) por quinquenio. Los valores o, como en el resto de tablas de este estudio, resultan del redondeo de decimales. (Fuente: elaboración propia).

Tabla 2. Tamaño VNP/VP por tipo.

\begin{tabular}{|c|c|c|r|r|r|r|r|}
\hline $\begin{array}{c}\text { CLASE Y } \\
\text { FECHA }\end{array}$ & \multirow{2}{*}{ TIPO } & \multirow{2}{*}{$\begin{array}{c}\text { \% DEL } \\
\text { TOTAL }\end{array}$} & \multicolumn{5}{|c|}{ DISTRIBUCIÓN DE TAMÃ̃OS } \\
\hline $\begin{array}{c}\text { VNP } \\
1940- \\
2009\end{array}$ & COL & 76,179 & 12,82 & 25,65 & 26,69 & 16,52 & 18,31 \\
\cline { 2 - 8 } & UNI & 2,046 & 7,97 & 5,26 & 5,43 & 4,79 & 76,55 \\
\hline $\begin{array}{c}\text { VP } \\
1940- \\
2009\end{array}$ & COL & 10,475 & 9,44 & 26,79 & 30,25 & 25,73 & 7,79 \\
\cline { 2 - 8 } & UNI & 0,597 & 5,83 & 17,94 & 47,64 & 17,34 & 11,25 \\
\hline $\begin{array}{c}\text { VNP + VP } \\
2000- \\
2014\end{array}$ & COL + UNI & 100,000 & 15,37 & 24,56 & 25,33 & 16,35 & 18,39 \\
\hline
\end{tabular}

Distribución en \% de los tamaños de VP/VNP 1940-2010 y del total VP + VNP hasta 2014, entre rangos (R1-R5), según tipo (COL/UNI), indicando el \% que supone esa categoría sobre el total de registros procesados hasta 2014. (Fuente: elaboración propia).

Tabla 3. Tamaño VNP/VP por arcos.

\begin{tabular}{|c|l|r|r|r|r|r|r|}
\hline \multirow{2}{*}{$\begin{array}{c}\text { POSICIÓN } \\
\text { Y FECHA }\end{array}$} & \multirow{2}{*}{ TIPO } & \multirow{2}{*}{ \% DEL } & \multicolumn{5}{|c|}{ DISTRIBUCIÓN DE TAMAÑOS (\%) } \\
\cline { 3 - 8 } & & TOTAL & \multicolumn{1}{|c|}{ R1 } & \multicolumn{1}{c|}{ R2 } & \multicolumn{1}{c|}{ R3 } & \multicolumn{1}{c|}{ R4 } & \multicolumn{1}{c|}{ R5 } \\
\hline \multirow{4}{*}{$\begin{array}{c}\text { ARC NOR } \\
1940-2009\end{array}$} & VNP COL & 33,350 & 14,70 & 20,51 & 20,99 & 16,12 & 27,68 \\
\cline { 2 - 8 } & VNP UNI & 1,734 & 7,82 & 3,91 & 3,83 & 4,06 & 80,38 \\
\cline { 2 - 8 } & VP COL & 2,139 & 10,54 & 25,12 & 31,15 & 23,43 & 9,75 \\
\cline { 2 - 8 } & VP UNI & 0,150 & 7,29 & 16,30 & 33,29 & 16,67 & 26,45 \\
\hline \multirow{4}{*}{$\begin{array}{c}\text { ARC SUR } \\
1940-2009\end{array}$} & VNP COL & 42,830 & 11,36 & 29,65 & 31,13 & 16,84 & 11,02 \\
\cline { 2 - 8 } & VNP UNI & 0,312 & 8,82 & 12,74 & 14,32 & 8,84 & 55,27 \\
\cline { 2 - 8 } & VP COL & 8,336 & 9,16 & 27,22 & 30,01 & 26,33 & 7,29 \\
\cline { 2 - 8 } & VP UNI & 0,447 & 5,34 & 18,49 & 52,45 & 17,56 & 6,16 \\
\hline
\end{tabular}

Distribución en \% de los tamaños de VP/VNP 1940-2010, entre rangos (R1-R5), según posición (ARC NOR/ARC SUR) y tipo (COL/UNI), indicando el \% que supone esa categoría sobre el total de registros procesados hasta 2014. (Fuente: elaboración propia).
Tabla 4. Tamaño VP por período.

\begin{tabular}{|c|c|c|c|c|c|c|c|}
\hline \multirow{2}{*}{ PERÍODO } & \multirow{2}{*}{\begin{tabular}{|c|} 
ARCO Y \\
TIPO
\end{tabular}} & \multirow{2}{*}{$\begin{array}{l}\text { \% DEL } \\
\text { TOTAL }\end{array}$} & \multicolumn{5}{|c|}{ DISTRIBUCIÓN DE TAMAÑOS (\%) } \\
\hline & & & $\mathbf{R 1}$ & R2 & $\mathbf{R} 3$ & $\mathbf{R 4}$ & $\mathbf{R}_{5}$ \\
\hline \multirow{4}{*}{$\begin{array}{l}\text { P.I } \\
1940-1974\end{array}$} & $\begin{array}{l}\text { ARC NOR } \\
\text { COL VP }\end{array}$ & 1,150 & 15,72 & 31,39 & 25,07 & 16,37 & 11,45 \\
\hline & $\begin{array}{l}\text { ARC NOR } \\
\text { UNI VP }\end{array}$ & 0,119 & 8,40 & 20,22 & 35,75 & 11,01 & 24,62 \\
\hline & \begin{tabular}{|l|} 
ARC SUR \\
COL VP \\
\end{tabular} & 4,310 & 16,17 & 42,97 & 31,52 & 7,31 & 2,02 \\
\hline & \begin{tabular}{|l|} 
ARC SUR \\
UNI VP \\
\end{tabular} & 0,288 & 1,34 & 27,86 & 58,71 & 9,65 & 2,44 \\
\hline \multirow{4}{*}{$\begin{array}{l}\text { P.II } \\
1975-1989\end{array}$} & $\begin{array}{l}\text { ARC NOR } \\
\text { COL VP } \\
\end{array}$ & 0,256 & 1,40 & 19,93 & 41,88 & 23,66 & 13,12 \\
\hline & $\begin{array}{l}\text { ARC NOR } \\
\text { UNI VP }\end{array}$ & 0,002 & 11,54 & 3,85 & 15,38 & 11,54 & 57,69 \\
\hline & \begin{tabular}{|l} 
AR SUR \\
COL VP
\end{tabular} & 1,999 & 0,38 & 3,34 & 24,51 & 56,58 & 15,19 \\
\hline & \begin{tabular}{|l|} 
ARC SUR \\
UNI VP
\end{tabular} & 0,089 & 0,08 & 0,47 & 39,15 & 42,79 & 17,52 \\
\hline \multirow{4}{*}{$\begin{array}{l}\text { P.III } \\
1990-1999\end{array}$} & $\begin{array}{l}\text { ARC NOR } \\
\text { COL VP }\end{array}$ & 0,312 & 3,92 & 14,14 & 38,78 & 36,35 & 6,81 \\
\hline & $\begin{array}{l}\text { ARC NOR } \\
\text { UNI VP }\end{array}$ & 0,013 & 4,89 & 0,54 & 51,09 & 16,30 & 27,17 \\
\hline & \begin{tabular}{|l} 
AR SUR \\
COL VP
\end{tabular} & 1,134 & 1,21 & 10,68 & 32,48 & 43,22 & 12,41 \\
\hline & \begin{tabular}{|l|} 
ARC SUR \\
UNI VP
\end{tabular} & 0,064 & 30,93 & 2,90 & 47,15 & 15,90 & 3,11 \\
\hline \multirow{4}{*}{$\begin{array}{l}\text { P.IV } \\
2000-2009\end{array}$} & $\begin{array}{l}\text { ARC NOR } \\
\text { COL VP }\end{array}$ & 0,422 & 6,89 & 19,31 & 35,58 & 32,99 & 5,24 \\
\hline & $\begin{array}{l}\text { ARC NOR } \\
\text { UNI VP }\end{array}$ & 0,016 & 0,43 & 0,87 & 0,00 & 60,00 & 36,09 \\
\hline & $\begin{array}{l}\text { AR SUR } \\
\text { COL VP }\end{array}$ & o,893 & 5,03 & 25,66 & 31,91 & 28,88 & 8,53 \\
\hline & \begin{tabular}{|l|} 
ARC SUR \\
UNI VP \\
\end{tabular} & 0,005 & 1,27 & 1,27 & 1,27 & 43,04 & 53,16 \\
\hline \multirow{2}{*}{$\begin{array}{l}\text { TOTAL } \\
1940-2009\end{array}$} & COL VP & 10,475 & 9,44 & 26,79 & 30,25 & 25,73 & 7,79 \\
\hline & UNI VP & 0,597 & 5,83 & 17,94 & 47,64 & 17,34 & 11,25 \\
\hline
\end{tabular}

Distribución en \% de los tamaños de VP 1940-2010 entre rangos (R1-R5), según período (P.I-P.IV), arco de posición (ARC NOR/ARC SUR) y tipo (COL/UNI), indicando el \% que supone esa categoría sobre el total de registros procesados hasta 2014. (Fuente: elaboración propia). 
Tabla 5. Tamaño VNP/VP por sectores.

\begin{tabular}{|c|c|c|c|c|c|c|c|}
\hline \multicolumn{8}{|c|}{ ARC NOR. COR 1. COL. 1940-2009 } \\
\hline \multirow{2}{*}{$\begin{array}{c}\text { ARCO/ } \\
\text { CORONA/ } \\
\text { SECTOR }\end{array}$} & \multirow{2}{*}{ TIPO } & \multirow{2}{*}{$\begin{array}{c}\% \\
\text { TOTAL }\end{array}$} & \multicolumn{5}{|c|}{ DISTRIBUCIÓN DE TAMAÑOS (\%) } \\
\hline & & & R1 & R2 & R3 & R4 & $\mathbf{R}_{5}$ \\
\hline \multirow{2}{*}{$\begin{array}{l}\text { ARC NOR } \\
\text { COR } 1 \\
\text { NO1 }\end{array}$} & \begin{tabular}{|l|} 
VNP COL \\
1940-2009 \\
\end{tabular} & 1,549 & 28,22 & 20,47 & 15,03 & 9,75 & 26,53 \\
\hline & \begin{tabular}{|l|} 
VP COL \\
$1940-2009$
\end{tabular} & 0,030 & 2,99 & 2,07 & 27,13 & 14,71 & 53,10 \\
\hline \multirow{2}{*}{$\begin{array}{l}\text { ARC NOR } \\
\text { COR } 1 \\
\text { N1 }\end{array}$} & \begin{tabular}{|l|} 
VNP COL \\
$1940-2009$ \\
\end{tabular} & 3,696 & 20,63 & 14,45 & 15,33 & 11,71 & 37,88 \\
\hline & \begin{tabular}{|l|} 
VP COL \\
$1940-2009$
\end{tabular} & 0,075 & 1,19 & 3,93 & 22,14 & 34,31 & 38,43 \\
\hline \multirow{2}{*}{$\begin{array}{l}\text { ARC NOR } \\
\text { COR } 1 \\
\text { NE1 }\end{array}$} & \begin{tabular}{|l|} 
VNP COL \\
$1940-2009$ \\
\end{tabular} & 2,739 & 21,31 & 15,47 & 16,05 & 11,44 & 35,73 \\
\hline & \begin{tabular}{|l|} 
VP COL \\
$1940-2009$
\end{tabular} & 0,108 & 1,15 & 8,97 & 35,83 & 30,13 & 23,91 \\
\hline \multirow{2}{*}{$\begin{array}{l}\text { ARC NOR } \\
\text { COR 1 } \\
\text { 1940-2009 }\end{array}$} & VNP COL & 7,984 & 22,34 & 15,97 & 15,52 & 11,24 & 34,94 \\
\hline & VP COL & 0,213 & 1,42 & 6,22 & 29,76 & 29,44 & 33,16 \\
\hline
\end{tabular}

\begin{tabular}{|c|c|c|c|c|c|c|c|}
\hline \multicolumn{8}{|c|}{ ARC SUR. COR 2. COL. 1940 - 2009} \\
\hline \multirow{2}{*}{$\begin{array}{c}\text { ARCO/ } \\
\text { CORONA/ } \\
\text { SECTOR }\end{array}$} & \multirow{2}{*}{ TIPO } & \multirow{2}{*}{$\begin{array}{c}\% \\
\text { TOTAL }\end{array}$} & \multicolumn{5}{|c|}{ DISTRIBUCIÓN DE TAMAÑOS (\%) } \\
\hline & & & R1 & $\mathbf{R 2}$ & $\mathbf{R}_{3}$ & R4 & $\mathbf{R 5}$ \\
\hline \multirow{2}{*}{$\begin{array}{l}\text { ARC SUR } \\
\text { COR } 2 \\
\text { E2 }\end{array}$} & \begin{tabular}{|l|} 
VNP COL \\
$1940-2009$ \\
\end{tabular} & 5,244 & 10,28 & 29,47 & 38,12 & 12,31 & 9,82 \\
\hline & \begin{tabular}{|l|} 
VP COL \\
$1940-2009$ \\
\end{tabular} & 2,501 & 17,56 & 38,60 & 29,71 & 9,37 & 4,76 \\
\hline \multirow{2}{*}{$\begin{array}{l}\text { ARC SUR } \\
\text { COR } 2 \\
\text { SE2 }\end{array}$} & \begin{tabular}{|l|} 
VNP COL \\
$1940-2009$ \\
\end{tabular} & 4,155 & 18,56 & 41,55 & 22,08 & 13,79 & 4,03 \\
\hline & \begin{tabular}{|l|} 
VP COL \\
$1940-2009$ \\
\end{tabular} & 1,788 & 3,20 & 16,70 & 26,63 & 44,74 & 8,73 \\
\hline \multirow{2}{*}{$\begin{array}{l}\text { ARC SUR } \\
\text { COR } 2 \\
\text { S2 }\end{array}$} & \begin{tabular}{|l} 
VNP COL \\
$1940-2009$ \\
\end{tabular} & 2,679 & 13,26 & 30,58 & 28,36 & 19,92 & 7,89 \\
\hline & \begin{tabular}{|l|} 
VP COL \\
$1940-2009$ \\
\end{tabular} & 1,399 & 4,20 & 18,18 & 29,07 & 39,55 & 9,00 \\
\hline \multirow{2}{*}{$\begin{array}{l}\text { ARC SUR } \\
\text { COR } 2 \\
\text { SO2 }\end{array}$} & \begin{tabular}{|l|} 
VNP COL \\
$1940-2009$ \\
\end{tabular} & 7,142 & 13,56 & 35,13 & 33,88 & 11,80 & 5,63 \\
\hline & \begin{tabular}{|l|} 
VP COL \\
$1940-2009$ \\
\end{tabular} & 1,025 & 8,28 & 28,67 & 43,10 & 12,27 & 7,68 \\
\hline \multirow{2}{*}{$\begin{array}{l}\text { ARC SUR } \\
\text { COR } 2 \\
1940-2009\end{array}$} & VNP COL & 19,220 & 13,70 & 34,34 & 31,71 & 13,50 & 6,74 \\
\hline & VP COL & 6,711 & 9,54 & 27,00 & 30,80 & 25,52 & 7,15 \\
\hline
\end{tabular}

\begin{tabular}{|c|c|c|c|c|c|c|c|}
\hline \multicolumn{8}{|c|}{ ARC SUR. COR 1. COL. 1940-2009 } \\
\hline \multirow{2}{*}{$\begin{array}{c}\text { ARCO/ } \\
\text { CORONA/ } \\
\text { SECTOR }\end{array}$} & \multirow{2}{*}{ TIPO } & \multirow{2}{*}{$\begin{array}{c}\% \\
\text { TOTAL }\end{array}$} & \multicolumn{5}{|c|}{ DISTRIBUCIÓN DE TAMAÑOS (\%) } \\
\hline & & & R1 & R2 & R3 & R4 & R5 \\
\hline \multirow{2}{*}{$\begin{array}{l}\text { ARC SUR } \\
\text { COR } 1 \\
\text { E1 }\end{array}$} & $\begin{array}{l}\text { VNP COL } \\
1940-2009\end{array}$ & 2,546 & 7,72 & 11,12 & 17,11 & 15,44 & 48,62 \\
\hline & $\begin{array}{l}\text { VP COL } \\
1940-2009\end{array}$ & 0,049 & 0,99 & 0,99 & 36,07 & 7,78 & 54,17 \\
\hline \multirow{2}{*}{$\begin{array}{l}\text { ARC SUR } \\
\text { COR } 1 \\
\text { SE1 }\end{array}$} & $\begin{array}{l}\text { VNP COL } \\
\text { 1940-2009 }\end{array}$ & 1,419 & 10,27 & 17,50 & 24,90 & 22,88 & 24,45 \\
\hline & \begin{tabular}{|l|} 
VP COL \\
$1940-2009$
\end{tabular} & 0,043 & 5,15 & 31,56 & 22,87 & 33,66 & 6,76 \\
\hline \multirow{2}{*}{$\begin{array}{l}\text { ARC SUR } \\
\text { COR } 1 \\
\text { S1 }\end{array}$} & $\begin{array}{l}\text { VNP COL } \\
1940-2009\end{array}$ & 1,683 & 9,85 & 21,82 & 31,16 & 22,69 & 14,48 \\
\hline & $\begin{array}{l}\text { VP COL } \\
1940-2009\end{array}$ & 0,052 & 4,27 & 9,73 & 43,73 & 34,27 & 8,00 \\
\hline \multirow{2}{*}{$\begin{array}{l}\text { ARC SUR } \\
\text { COR } 1 \\
\text { SO1 }\end{array}$} & $\begin{array}{l}\text { VNP COL } \\
1940-2009\end{array}$ & 3,042 & 16,90 & 19,11 & 25,08 & 21,43 & 17,48 \\
\hline & $\begin{array}{l}\text { VP COL } \\
1940-2009\end{array}$ & 0,065 & 5,87 & 22,20 & 39,81 & 26,68 & 5,44 \\
\hline \multirow{2}{*}{$\begin{array}{l}\text { ARC SUR } \\
\text { COR } 1 \\
1940-2009\end{array}$} & VNP COL & 8,689 & 11,76 & 17,03 & 23,89 & 20,16 & 27,16 \\
\hline & VP COL & 0,208 & 4,18 & 16,05 & 36,42 & 25,57 & 17,78 \\
\hline
\end{tabular}

\begin{tabular}{|c|c|c|c|c|c|c|c|}
\hline \multicolumn{8}{|c|}{ ARC NOR. COR 3. COL. 1940 - 2009} \\
\hline \multirow{2}{*}{$\begin{array}{c}\text { ARCO/ } \\
\text { CORONA/ } \\
\text { SECTOR }\end{array}$} & \multirow{2}{*}{ TIPO } & \multirow{2}{*}{$\begin{array}{c}\% \\
\text { TOTAL }\end{array}$} & \multicolumn{5}{|c|}{ DISTRIBUCIÓN DE TAMAÑOS (\%) } \\
\hline & & & $\mathbf{R 1}$ & R2 & $\mathbf{R}_{\mathbf{3}}$ & R4 & $\mathbf{R}_{\mathbf{5}}$ \\
\hline \multirow{2}{*}{$\begin{array}{l}\text { ARC NOR } \\
\text { COR } 3 \\
\mathrm{NO}_{3}\end{array}$} & $\begin{array}{l}\text { VNP COL } \\
1940-2009\end{array}$ & 0,930 & 11,24 & 29,50 & 18,79 & 15,18 & 25,30 \\
\hline & $\begin{array}{l}\text { VP COL } \\
1940-2009\end{array}$ & 0,001 & 10,00 & 90,00 & 0,00 & 0,00 & 0,00 \\
\hline \multirow{2}{*}{$\begin{array}{l}\text { ARC NOR } \\
\text { COR } 3 \\
\mathrm{~N}_{3}\end{array}$} & \begin{tabular}{|l|} 
VNP COL \\
$1940-2009$ \\
\end{tabular} & 6,158 & 10,01 & 19,56 & 19,86 & 22,60 & 27,98 \\
\hline & $\begin{array}{l}\text { VP COL } \\
1940-2009\end{array}$ & 0,483 & 9,36 & 33,36 & 35,12 & 17,87 & 4,29 \\
\hline \multirow{2}{*}{$\begin{array}{l}\text { ARC NOR } \\
\text { COR } 3 \\
\text { NE3 }\end{array}$} & $\begin{array}{l}\text { VNP COL } \\
\text { 1940-2009 }\end{array}$ & 5,899 & 7,25 & 17,36 & 26,62 & 22,54 & 26,23 \\
\hline & $\begin{array}{l}\text { VP COL } \\
1940-2009 \\
\end{array}$ & 0,559 & 8,44 & 29,77 & 29,51 & 26,55 & 5,74 \\
\hline \multirow{2}{*}{$\begin{array}{l}\text { ARC NOR } \\
\text { COR } 3 \\
1940-2009\end{array}$} & VNP COL & 12,987 & 8,84 & 19,27 & 22,85 & 22,04 & 26,99 \\
\hline & VP COL & 1,042 & 8,87 & 31,47 & 32,09 & 22,51 & 5,06 \\
\hline
\end{tabular}

\begin{tabular}{|c|c|c|c|c|c|c|c|}
\hline \multicolumn{8}{|c|}{ ARC NOR. COR 2. COL. 1940-2009 } \\
\hline \multirow{2}{*}{$\begin{array}{c}\text { ARCO/ } \\
\text { CORONA/ } \\
\text { SECTOR }\end{array}$} & \multirow{2}{*}{ TIPO } & \multirow{2}{*}{$\begin{array}{c}\% \\
\text { TOTAL }\end{array}$} & \multicolumn{5}{|c|}{ DISTRIBUCIÓN DE TAMAÑOS (\%) } \\
\hline & & & R1 & R2 & R3 & $\mathbf{R} 4$ & $\mathbf{R}_{5}$ \\
\hline \multirow{2}{*}{$\begin{array}{l}\text { ARC NOR } \\
\text { COR } 2 \\
\text { NO2 }\end{array}$} & \begin{tabular}{|l} 
VNP COL \\
$1940-2009$ \\
\end{tabular} & 0,097 & 6,72 & 22,23 & 17,87 & 12,08 & 41,10 \\
\hline & \begin{tabular}{|l} 
VP COL \\
$1940-2009$
\end{tabular} & 0,171 & 6,85 & 31,01 & 22,94 & 25,36 & 13,83 \\
\hline \multirow{2}{*}{$\begin{array}{l}\text { ARC NOR } \\
\text { COR } 2 \\
\text { N2 }\end{array}$} & \begin{tabular}{|l} 
VNP COL \\
$1940-2009$
\end{tabular} & 5,521 & 20,61 & 27,45 & 21,90 & 11,73 & 18,31 \\
\hline & $\begin{array}{l}\text { VP COL } \\
1940-2009\end{array}$ & 0,480 & 14,79 & 20,82 & 33,88 & 23,20 & 7,31 \\
\hline \multirow{2}{*}{$\begin{array}{l}\text { ARC NOR } \\
\text { COR } 2 \\
\text { NE2 }\end{array}$} & \begin{tabular}{|l|} 
VNP COL \\
$1940-2009$
\end{tabular} & 6,761 & 12,22 & 22,58 & 23,20 & 14,13 & 27,87 \\
\hline & \begin{tabular}{|l} 
VP COL \\
$1940-2009$ \\
\end{tabular} & 0,233 & 20,35 & 18,54 & 28,69 & 21,12 & 11,30 \\
\hline \multirow{2}{*}{$\begin{array}{l}\text { ARC NOR } \\
\text { COR } 2 \\
1940-2009\end{array}$} & VNP COL & 12,378 & 15,92 & 24,75 & 22,57 & 13,04 & 23,71 \\
\hline & VP COL & 0,884 & 14,72 & 22,19 & 30,39 & 23,07 & 9,63 \\
\hline
\end{tabular}

\begin{tabular}{|c|c|c|c|c|c|c|c|}
\hline \multicolumn{8}{|c|}{ ARC SUR. COR 3. COL. 1940 - 2009} \\
\hline \multirow{2}{*}{$\begin{array}{c}\text { ARCO/ } \\
\text { CORONA/ } \\
\text { SECTOR }\end{array}$} & \multirow{2}{*}{ TIPO } & \multirow{2}{*}{$\begin{array}{c}\% \\
\text { TOTAL }\end{array}$} & \multicolumn{5}{|c|}{ DISTRIBUCIÓN DE TAMAÑOS (\%) } \\
\hline & & & R1 & $\mathbf{R 2}$ & $\mathbf{R 3}$ & R4 & $\mathbf{R} 5$ \\
\hline \multirow{2}{*}{$\begin{array}{l}\text { ARC SUR } \\
\text { COR } 3 \\
E_{3}\end{array}$} & \begin{tabular}{|l|} 
VNP COL \\
1940-2009 \\
\end{tabular} & 3,926 & 6,08 & 25,68 & 32,30 & 25,63 & 10,30 \\
\hline & \begin{tabular}{|l|} 
VP COL \\
$1940-2009$ \\
\end{tabular} & 0,302 & 0,14 & 3,68 & 19,51 & 65,39 & 11,29 \\
\hline \multirow{2}{*}{$\begin{array}{l}\text { ARC SUR } \\
\text { COR } 3 \\
\text { SE3 }\end{array}$} & $\begin{array}{l}\text { VNP COL } \\
1940-2009 \\
\end{array}$ & 2,589 & 14,94 & 20,53 & 37,73 & 19,80 & 7,01 \\
\hline & \begin{tabular}{|l} 
VP COL \\
$1940-2009$ \\
\end{tabular} & 0,366 & 13,55 & 36,30 & 29,77 & 17,45 & 2,92 \\
\hline \multirow{2}{*}{$\begin{array}{l}\text { ARC SUR } \\
\text { COR } 3 \\
\text { S3 }\end{array}$} & $\begin{array}{l}\text { VNP COL } \\
1940-2009 \\
\end{array}$ & 3,415 & 7,93 & 34,64 & 32,62 & 19,10 & 5,72 \\
\hline & \begin{tabular}{|l|} 
VP COL \\
$1940-2009$ \\
\end{tabular} & 0,475 & 6,97 & 39,68 & 28,96 & 19,27 & 5,11 \\
\hline \multirow{2}{*}{$\begin{array}{l}\text { ARC SUR } \\
\text { COR } 3 \\
\mathrm{SO}_{3}\end{array}$} & \begin{tabular}{|l|} 
VNP COL \\
$1940-2009$ \\
\end{tabular} & 4,991 & 6,29 & 38,02 & 36,11 & 13,94 & 5,64 \\
\hline & \begin{tabular}{|l|} 
VP COL \\
$1940-2009$ \\
\end{tabular} & 0,273 & 11,51 & 33,30 & 19,61 & 27,56 & 8,02 \\
\hline & & & & & & & \\
\hline \multirow{2}{*}{$\begin{array}{l}\text { ARC SUR } \\
\text { COR } 3 \\
1940-2009\end{array}$} & VNP COL & 14,921 & 8,11 & 30,96 & 34,59 & 19,21 & 7,12 \\
\hline & VP COL & 1,416 & 8,09 & 29,90 & 25,35 & 30,24 & 6,43 \\
\hline
\end{tabular}

Distribución en \% de los tamaños de la mayoritaria categoría COL promovida entre 1940-2010, tanto en VP como VNP, entre rangos (R1-R5), según posición, diferenciando arcos (ARC NOR/ARC SUR), coronas (COR 1, 2, 3) y sectores (NO, N, NE, E, SE, S y SO). (Fuente: elaboración propia). 


\section{ABREVIATURAS UTILIZADAS}

ARCOS: 2 arcos de posición urbana: ARC NOR (Arco Norte) y ARC SUR (Arco Sur).

SECTORES: dentro del ARC NOR, tres sectores, en sentido horario NO (Noroeste), N (Norte) y NE (Noreste); en el ARC SUR, cuatro sectores, en sentido horario E (Este), SE (Sureste), S (Sur) y SO (Suroeste).

CORONAS: 3 coronas de posición urbana: COR 1 (Cibeles-radio $3 \mathrm{~km})$, COR $2(3-6 \mathrm{~km})$ y COR 3 (6 km-límite término municipal).

PERÍODOS: 4 períodos: P.I (Q1-Q7), P.II (Q8-Q10), P.III (Q.11-Q.12) y P.IV (Q.13-Q14). A su vez el P.I se subdivide en los siguientes subperíodos: P.I.1 (Q1-Q3), P.I.2 (Q4-Q5) y P.I.3 (Q6-Q7).

QUINQUENIOS: 14 quinquenios entre 1940-2010: Q1 (1940-1944), Q2 (1945-1949), Q3 (1950-1954), Q4 (1955-1959), Q5 (1960-1964), Q6 (1965-1969), Q7 (1970-1974), Q8 (1975-1979), Q9 (1980-1984), Q10 (1985-1989), Q11 (1990-1994), Q12 (1995-1999), Q13 (2002004) y Q14 (2005-2009).

CLASES: 2 clases de vivienda: VP (Vivienda Pública) y VNP (Vivienda No Pública).

TIPOS: 2 tipos edificatorios vivienda: COL (Colectiva) y UNI (Unifamiliar).

RANGOS: 5 rangos de tamaños del parque de vivienda, en superficie útil: R1 (< $\left.45 \mathrm{~m}^{2} \mathrm{u}\right), \mathrm{R} 2$ (45-6o m²u), R3 (6o-75 m²u), R4 (75-9o $\left.\mathrm{m}^{2} \mathrm{u}\right)$ y $\mathrm{R} 5\left(>90 \mathrm{~m}^{2} \mathrm{u}\right)$.

\section{REFERENCIAS}

(1) Barreiro, P. (1991). Casas Baratas: la vivienda social en Madrid 19oo-1939. Madrid: Colegio Oficial de Arquitectos de Madrid.

(2) Valenzuela, M. (1974). Iniciativa oficial y crecimiento urbano en Madrid (1939-1973). Estudios geográficos, 135: 593655 .

(3) Terán, F. (1992). Madrid, p. 52. Madrid: Mapfre.

(4) Moya, L. (1983). Barrios de promoción oficial. Madrid 1939-1976. Madrid: Colegio Oficial de Arquitectos de Madrid.

(5) Leal, J., Domínguez, M. (2008). Transformaciones económicas y segregación social en Madrid. Ciudad y Territorio, Estudios Territoriales, 158: 703-726.

(6) Roch, F. (2009). Morfología, deterioro urbano y precio de la vivienda en Madrid. Ciudades, 12: 171-196.

(7) Secchi, B. (2015). La ciudad de los ricos y la ciudad de los pobres. Madrid: Catarata.

(8) Moya, L. (Ed.). (2007). Vivienda Reducida. Madrid: Mairea.

(9) Sambricio, C. (Ed.). (2009). La Vivienda Protegida. Historia de una necesidad. Madrid: Ministerio de la Vivienda.

(10) Moya, L. (2015). Los antecedentes franquistas de la política de la vivienda social. Cuadernos de Investigación Urbanística, CIUR, 100: 73-76.

(11) Moya, L., F. Salgado, C. (2012). La Operación de Remodelación de Barrios de Madrid en los '80': un antecedente de regeneración urbana integral con participación ciudadana en el campo de la Vivienda Social. En Pinzello, I. (Coord.), Verso una nuova politica della casa. Politiche pubbliche e modelli abitativi in Italia e in Spagna (pp. 117-137). Milán: Franco Angeli Edizioni.

(12) Moya, L., F. Salgado, C. (2014, 26 de febrero). La forma urbana de la periferia y los conjuntos de vivienda pública [VP]. Madrid 1940-2010. En Montaner, J. M. (Ed.), Actas I Congreso Internacional de Vivienda Colectiva Sostenible Barcelona 25, 26 y 27 de febrero de 2014 (pp. 244-249). Barcelona: ETSAB, Universidad Politécnica de Cataluña.

(13) Vinuesa, J. (2013). El festín de la vivienda: auge y caída del negocio inmobiliario en España. Madrid: Díaz \& Pons.

(14) Paquot, T. (Ed.). (2008). Banlieues. Une anthologie, Lausanne : Presses polytechniques et universitaires romandes.

(15) Hernández, A., Matesanz, A., García, C. [Dir.]. (2015). Atlas de barrios vulnerables de España: 12 ciudades 1991-20012006. Madrid: Instituto Juan de Herrera. 